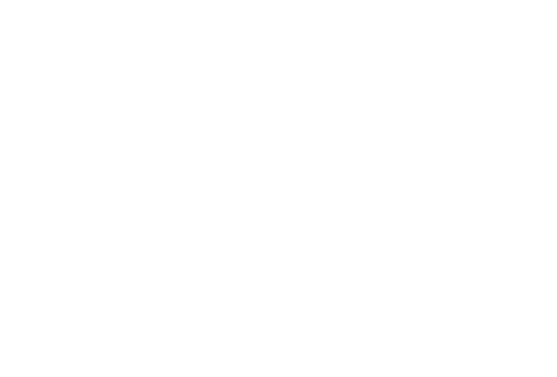

Annu. Rev. Entomol. 2010. 55:505-20

First published online as a Review in Advance on September 9, 2009

The Annual Review of Entomology is online at ento.annualreviews.org

This article's doi:

10.1146/annurev-ento-112408-085342

Copyright (C) 2010 by Annual Reviews. All rights reserved

$0066-4170 / 10 / 0107-0505 \$ 20.00$

\section{Physical Ecology of Fluid Flow Sensing in Arthropods}

\author{
Jérôme Casas ${ }^{1}$ and Olivier Dangles ${ }^{2}$ \\ ${ }^{1}$ University of Tours, Institut de Recherche sur la Biologie de l'Insecte, \\ IRBI UMR CNRS 6035, 37200 Tours, France; email: jerome.casas@univ-tours.fr \\ ${ }^{2}$ IRD, UR 072, LEGS, UPR 9034, CNRS 91198 Gif-sur Yvette Cedex, \\ France and Université Paris-Sud 11, 91405 Orsay Cedex, France; \\ email: Olivier.Dangles@legs.cnrs-gif.fr
}

\section{Key Words}

sensory ecology, hairs, mechanosensors, sand flow, biomimetic MEMS, viscous flow

\begin{abstract}
Terrestrial and aquatic arthropods sense fluid flow in many behavioral and ecological contexts, using dedicated, highly sensitive mechanosensory hairs, which are often abundant. Strong similarities exist in the biomechanics of flow sensors and in the sensory ecology of insects, arachnids, and crustaceans in their respective fluid environments. We extend these considerations to flow in sand and its implications for flow sensing by arthropods inhabiting this granular medium. Finally, we highlight the need to merge the various findings of studies that have focused on different arthropods in different fluids. This could be achieved using the unique combination, for sensory ecology, of both a workable and well-accepted mathematical model for hair-based flow sensing, both in air and water, and microelectronic mechanical systems microtechnology to tinker with physical models.
\end{abstract}




\section{INTRODUCTION}

Physical ecology: a sister science to chemical ecology concerned with the physical interactions between an organism and its biotic and abiotic environments
Studies of flow sensing have often taken divergent approaches for arthropods living in aquatic and terrestrial environments. Seminal work on the morphology and behavior of insect larvae in relation to current speeds of running water in the nineteenth century and until the middle of the twentieth century formed the basis for subsequent studies on flow sensing in aquatic insects $(34,71)$. Such studies were, and still are, carried out under the ecological conditions, at a macroscale level, of the flow experienced by the organisms. For smaller copepods living in the open waters, studies from the 1960s onward have provided a clear picture of flow sensing systems ranging from microscale three-dimensional perception fields to population dynamics (46). There have been, however, few comprehensive studies on the neurophysiology and neuroethology of aquatic insects and copepods (but see the notable results with copepods in References 27, 28, 44, 50, 80, 87, and 88 , among others). The difficulties associated with electrophysiological studies on aquatic organisms, in particular the smallest ones, may explain the paucity of such studies.

By contrast, studies investigating fluid flow sensing in terrestrial arthropods began later but quickly diverged from the natural environment and ecologically relevant stimuli, not only to the benefit of neuroscience $(2,9,33,60,61,64$, $74)$, but to the detriment of ecological and evolutionary science (15). This departure, still evident today despite substantial advances in the domain of neuroecology $(24,52,62,67)$, is more difficult to explain.

There is no recent overview of the physical ecology of flow sensing in arthropods highlighting the similarities and differences between arthropods living on land and in water. The only review of this type, written from a neuroethological standpoint, is the German monograph by Tautz (74). The aim of this review is therefore to provide a comprehensive overview of the physical aspects of flow sensing using hairs in arthropods, ranging from the characterization of the fluid forces acting on a hair to the ecological context in which such stimuli are generated. We consider flow sensing in air, water, and sand, the last behaving like both a liquid and a solid. We chose an approach based on the physics of the environment and across arthropods, including copepods, to make our points about analogies and differences. Physical ecology, a younger sister science of chemical ecology, must deal with these matters as deeply as chemical ecology must deal with the biochemistry of secondary compounds or the photochemistry of pigments, for example. Physics is to physical ecology what chemistry is to chemical ecology, and we opted for a presentation of how an understanding of the constraints imposed by physics influences morphology, physiology, behavior, and ecology. Finally, this review does not cover the mechanics of the hair itself, which was the topic of a recent comprehensive review (38), or the neurosensory aspects of signal detection and identification, or olfaction, which is a physicochemical topic with a strong component of fluid dynamics.

\section{DIVERSITY OF FLOW SENSORS}

\section{Diversity of Structures}

Most arthropods are hairy animals. Many of these hairs are mechanoreceptors, some of which are chemosensory $(3,7)$. In several groups, such as crustaceans, arachnids, and insects, many of these mechanoreceptors serve as fluid flow sensors. These so-called filiform hairs in insects, trichobothria in arachnids, and flow sensilla in crustaceans detect air, sand, or water particle displacements and are among the most sensitive sensory organs in the animal world. Cricket filiform hairs can perceive air movements at speeds as low as $0.03 \mathrm{~mm} \mathrm{~s}^{-1}$ (68), whereas calanoid copepods can detect water stimulus displacements as small as $10 \mathrm{~nm}$ (80). All fluid flow sensors have a similar design and represent hair-like structures. Suspended in a cuticular socket, the hair shaft functions as a lever, which responds to fluid viscous forces in the surrounding medium. The movement of the inner end of the hair shaft during 
deflection triggers one or several sensory neurons that transmit signals indicating the velocity, acceleration, and direction of the flow to the central nervous system to induce appropriate behavior (38). Directional sensitivity is essential to both terrestrial and aquatic arthropods to localize the source of air fluctuation and has been the focus of many studies on biomechanics and neuronal processing (see Reference 40 and references therein).

Despite this overall similarity, fluid flow sensors are not homologous among or within arthropod groups. For example, arthropod fluid flow sensors differ in the location of flowsensing hairs on the animal's body. In orthopterid insects, the location of flow-sensing hairs is mainly at the rear end of the abdomen (specifically on the cerci), thereby providing useful flow information about predators approaching from behind. In spiders and crayfish, they are mainly found on the front (forelegs and antennules) and posterior (hindlegs and tailfan) appendages of the body, serving as detectors of both prey and predators $(3,74)$. In marine copepods, they are located on the antennules, allowing these animals to scan the environment at a distance from their bodies (27). The shapes of the structures bearing the flow sensors are therefore also different, ranging from the conic cerci of crickets to the rather flat crayfish tailfan, with significant implications for fluid flow patterns and sensing (see Boundary Layer and Hair Lengths, below). The shape of the hair shaft may also differ, from strictly filiform (e.g., orthopterid insects), to flattened or feathery at the tip, as in some spiders and crustaceans.

Flow sensors also differ in number and shaft length, both within (e.g., during ontogeny) and among species. Flow-sensing hair length thus varies between approximately 10 and $2000 \mu \mathrm{m}$, with extreme values reaching 2500 and $3000 \mu \mathrm{m}$ in arachnids and crickets, respectively $(38,53$, $80,83)$. The number of flow sensors inserted into the cuticle on the animal's body surface is more variable: Some early stages of cockroaches bear few filiform hairs on their cerci, whereas a Nembobius sylvestris adult has more than 300 (20). Thus, the morphology, number, and arrangement of flow-sensing hairs are highly diverse features. In this review, we explain this variation based on physical insights. We restrict our analysis to hairs moving in air, the study of which has been marked by significant advances. Fluid movement around and through arrays of hairs on aquatic animals $(12,48)$ has been extensively studied, but mostly in terms of food acquisition or olfaction.

\section{Boundary Layer and Hair Lengths}

The nature of the flow and its interaction with the hairs is dependent on the shape of the sensory platform on which sensors are inserted (20). This relationship has been demonstrated for chemical and fluid motion sensors found in crustaceans, arachnids, and insects, in which the sensory platform affects the hydrodynamics of the fluid flow across its surface $(3,20,38,48$, 72). The variation in diameter of the conic cercus of crickets modifies the pattern of fluid velocity around the sensory platform, for example (20). Thus, hairs are subject to higher air flow amplitudes at the base than at the apex of the cercus. The radial position of hairs around the cercus can also affect the cricket's perception of the direction of incoming danger. Hairs of the same length but positioned at different locations on the cercus move with different amplitudes as they experience different longitudinal and transverse forces over their shafts (38, 72). Thus, the platform on which hairs are positioned determines the extent of the boundary flow.

What are the characteristics of boundary flow around the platform in which hairs are immersed? First, owing to the low flow velocity and small size of hairs and appendages, the Reynolds number (Re) is quite low, below 1 . This is a typical feature of viscous flow. Second, the fact that system approach analyses use sine waves of many frequencies and the assumption that flying wasps are the main predators explain why nearly all experiments and models of the cricket air-flow-sensing system use sinusoidal stimuli. Because the viscous boundary flow is oscillatory, certain
Reynolds number $(\mathrm{Re})$ : ratio of the inertia forces $(U$, speed of the organism or flow, times $L$, characteristic length of the organism) and the viscous forces ( $v$, kinematic viscosity)

Viscous flow: flow for which $\operatorname{Re} \ll 1$, in which the viscous forces dominate and the inertial forces are negligible 
Torque: a force that produces rotation

Viscous coupling: viscosity-mediated coupling between hairs at low $\operatorname{Re}$

Canopy flow: flow in an array of obstacles counterintuitive physical phenomena occur (72). In a nonoscillatory boundary layer, the flow velocity increases monotonically from zero at the surface to the far field velocity. For oscillatory flows, the peak of the amplitude is a short distance above the boundary. This distance is a function of the frequency of the flow oscillations and of the geometry of the substrate. For the cercus of Nemobius sylvestris and for stimulus frequencies in the range of those produced by flying wasps $(150-170 \mathrm{~Hz})$, the velocity peak is at $300 \mu \mathrm{m}(20)$. Thus, a hair of $1 \mathrm{~mm}$ or shorter is fully immersed in this complex flow. Movement of the hair results from the summation of torque acting along its length. This relationship also explains why hairs longer than a few millimeters are not frequently observed: Their length correlates with the thickness of the boundary layer, itself a function of the flow frequency (38). These considerations show that to some extent the diversity of hair morphology can be explained by the physical interactions between the hair and its fluid environment.

\section{Emergent Properties of Arrays of Hairs}

For years, the study of fluid flow sensors was based exclusively on the biomechanics of a single hair. However, many arthropods have body regions densely populated with sensory hairs, which exert effects on each other. The influence of neighboring hairs depends on the flow characteristics (frequency and speed), hair characteristics (length and directivity), and distances between hairs. In many cases, the fluid forces acting on a hair are determined by the flow induced by other moving hairs. This so-called viscous coupling is the topic of several recent studies, as detailed below. The coupling between hairs could be viewed as beneficial, taking into account the need for a set of responses to trigger an appropriate behavioral response. Alternatively, it could be seen as a hindrance, with hairs interfering with each other and causing the independence of the sensors to be lost. For example, it has been proposed that viscous coupling between air-flow-sensing hairs may be undesirable for predators (e.g., spiders) in the detection of their prey but desirable for prey (e.g., cockroaches) in the detection of their predators (38). Whereas predators might need independent information from many hairs for accurate localization of their target, prey may need to have many hairs moving simultaneously to trigger an effective escape.

In a combined experimental and theoretical study, long and short hairs placed near each other seemed to exert more effects on each other than did hairs of similar length (4). Such interactions could not be detected by analyzing the spatial point patterns made by the cuticular sockets, but a uniform pattern in the arrangement of long hairs, also indicative of a repulsion mechanism, was identified (20). Analytical modeling by Bathellier et al. (4) and computational modeling based on observed hair positioning by Cummins et al. (14) highlight noticeable effects of a hair on its nearest neighbor at distances of between 30 and 100 times the hair diameter, a considerable distance. Further studies are needed to continue this fascinating work, in terms of both experimentation and modeling. Extending these ideas from tandem hairs to full arrays of hairs represents yet another challenge. Studies of urban and forest canopy flows suggest that fluid flow phenomena such as dead zones and recirculation swirls within the array or velocity profiles with peaks above the array, in contrast to the peak at mid-height for a long hair on a flat plate, could be expected, at least at high velocities (31). Therefore, the functional unit is often a group of hairs, and fluid flow sensing most likely uses the emergent properties of the group of hairs as a whole. Thus, the study of viscous coupling between hairs will offer potential insight into biophysical mechanisms underlying the long unanswered question of why insects are loaded with so many hairs.

\section{Functional Significance of Morphological Diversity}

Structure-function relationships between mechanosensor properties and their sensory roles have been the subject of extensive studies, 
which have been comprehensively reviewed for most arthropod groups $(2,3,68)$. Briefly, the number of hairs and variation in length are key features of most fluid-flow-sensing systems, as they determine the variability of both the intensity and range of frequencies of a flow stimulus. Longer hairs are usually more sensitive to small flow displacements; marine copepod predators tend to have longer sensilla than herbivores, making them more sensitive to small flow displacements (27). In some cases, hair flattening or feathering can increase the drag force acting on the shaft and therefore its sensitivity (38). Further studies of fluid flow sensors incorporating ontogenetic and phylogenetic features may provide a deeper understanding of the causes and consequences of this diversity: The selection forces may change drastically over the course of an arthropod's lifetime. For example, in many groups such as copepods, the functional relevance of the extensive diversity of antennules and hair morphology, orientation, spacing, and degree of ornamentation is virtually unexplored $(7,27)$.

\section{ECOLOGICAL SIGNALS}

\section{Need for Ecological Signals}

Flow motion provides a wealth of ecologically relevant information in both terrestrial and aquatic habitats. Flow itself not only provides vital information to a wide variety of organisms, but its characteristics influence how animals send or process sensory information, such as in insect and crustacean chemical cue acquisition $(5,82,84,85)$. For example, flows characterized by high shear velocity (the shear stress per surface) and high Re relative to surface roughness reduce the ability of crabs to track clam scents (82). In addition, under conditions of weak or nonexistent flow, animals can facilitate food searching by generating currents themselves (21). Here, we focus on studies of purely fluid mechanical signals.

Fluid mechanical signals typically constitute the proximate cue for a wide variety of arthropod functions, including prey detection $(2,26,50)$, predator detection $(8,17,27,28$, $33)$, mating $(1,43)$, and flight control $(37,65)$. Even when no attractive or repellent chemical cues are associated with the flow cue, insects and crustaceans can choose to move in a direction related to the direction of flow (rheotaxis) or other targets. For example, many aquatic invertebrates orient their bodies and their locomotion to the direction of water flow around them $(44,71,86)$. Flow can serve as a directional cue in navigation, or orientation to flow can serve as a mechanism to reduce hydrodynamic forces $(59,71)$. For animals that are nocturnal or blind, or living in turbid waters, orientation to flow could be one of the rare sources of directional information. Krill species seem to use flow sensing in yet another social context, by schooling to use energy from the wake (61). The propulsion jet flow behind a shrimp is surrounded by a vortex ring, which a following shrimp can use to extract lift and forces during long-distance migration. A final example is the pioneering work of Tautz on the hair-based early-warning systems of caterpillars and their behavioral responses when attacked by wasps $(74,75)$. The broadband response of these filiform hairs to flight frequencies led to the recently discovered cascade in food webs: Caterpillars eat less, for example, when the attacked plants are visited by flying pollinators (76).

Despite the existence of many clear-cut examples of ecological signals, unnatural stimuli have been and are routinely applied to sensors, often in the form of sine waves. Such stimuli trigger neurophysiological responses, and the approach has deep roots in system analysis (30). The real difficulty lies in interpreting the outcome for higher levels of integration, that is, the implications of a spike train (63). For example, the basilar membrane in the vertebrate auditory system, but few cells in the auditory cortex, can respond to unnatural continuous pure tones (69). However, the rustling and crackling of predators creeping through the undergrowth, among other sounds, prove far more effective stimuli. The same is true when moving up from the retina, which responds to spots of bright 
light, to the visual cortex, for which edges at different orientations are much more effective. The concept of the grandmother-recognizing neuron, irrespective of the difficulties of its operative definition, offers a vivid illustration of these principles (69). Thus, without considering the ecological signals, much of the how and most of the why of a mechanical or behavioral response of a hair cannot be understood (47).

\section{Insights from Predator-Prey Interactions}

Because they are involved in many interspecific interactions, a sound ecological understanding of these flow sensors should incorporate the sensory aspects of both partners involved (16). Several integrated approaches to studying fluid flow sensing have included an analysis of both partners, especially in predator-prey interactions such as between spider and fly (2), bat and mantis (77), frog and spider (73), spider and cricket $(10,15,18,19)$, and herbivore and predator copepods (50). One of the central issues in our understanding of flow-mediated predator-prey interactions is timing, that is, the time available, between predator detection and capture, for the prey to carry out effective escape strategies. To address this issue, sensory ecologists have made considerable efforts in recording and reproducing flow movements that accompany predator attack or prey displacement and testing the associated response of prey or predators, respectively. For example, to determine the response of the mantis cercal system to signals generated by a flying attacking bat, Triblehorn \& Yager (77) trained a bat to fly consistently from a perch directly to the mantis. For studies in which predator behavior could not be manipulated, predator models have been designed. Suter (73) developed a model predator of a freeze-dried bullfrog simulating attacks on live spiders to evaluate the role of trichobothria in mediating the evasive responses to natural predators. Similarly, a piston simulating spider attacks on crickets was designed (18). Generally, most studies of flow-mediated predator-prey interactions demonstrated the importance of flow as essential information not only for successfully escaping from predators or attacking prey, but also in shaping the tactics used by both predators and prey. Spiders modify their hunting strategies depending on distance to the prey, and these strategies are constrained by the air flow displacements generated by the predators when running toward their prey (10, 18) (Figure 1). Similarly, predatory copepods may be capable of altering the strength of the hydrodynamic signals presented to potential prey by changing the magnitude of both fluid velocity and shear in the feeding current (8).

A recent example demonstrates the power of using an ecological signal to understand the design of a fluid-flow-sensing system and the danger of relying on unnatural stimuli in studies of predator-prey interactions. The use of continuous sine waves has enabled the scientific community to dissect the internal workings of the cricket black box, the terminal abdominal ganglion. This unnatural stimulus has also served as a useful tool in characterizing the biomechanical basis of hair response (38). For several decades, it was assumed that long hairs are triggered by the smallest amplitudes of a sine signal, at low frequencies, whereas the numerous short hairs react at higher amplitudes, at higher frequencies (53). This notion was based on the resonance frequency of hairs of different lengths, a concept intimately linked to oscillatory input. However, this view has now been superseded by one based on transient signals of short duration, such as those produced by approaching spiders and detected by escaping crickets $(10,18)$. The latest study shows that when pulse functions are used that closely mimic the characteristics of the launch of an attack by a spider, rather than sine waves, the short hairs react first (45).

\section{COPEPODOLOGY FOR ENTOMOLOGISTS}

Whereas it is challenging to visualize flow stimuli in the air and correlate behavior with stimulus patterns, visualization of flow tracers in water is much easier, and crustaceans offer a highly 


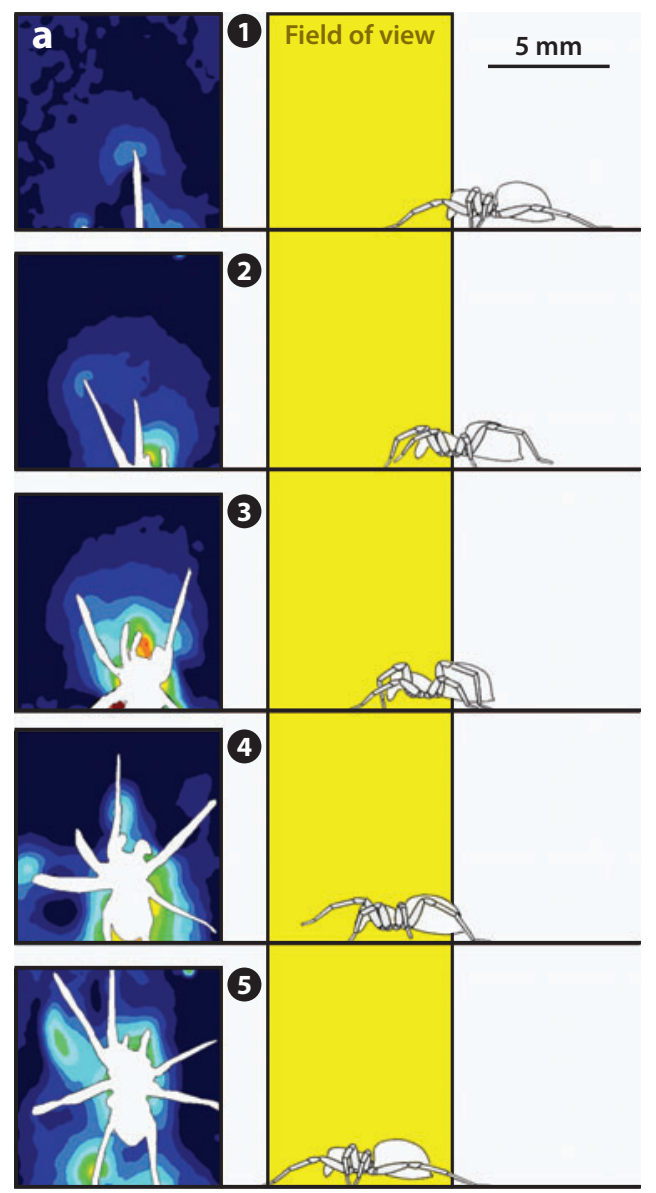

Figure 1

(a) Flow field and (b) close-up view of the flow around a running Pardosa spider (running at $10 \mathrm{~cm} \mathrm{~s}^{-1}$ ) measured using particle image velocimetry. The sequence in panel $a$ highlights the pockets of high air flow velocity created by leg strokes superposed on the air movements created by the body trunk movement. The schematic highlights the relative position of legs to body trunk. The zone of flow velocities above the measurable range is in black. (c) Flow velocity upstream of running spiders. The observed speeds (mean and standard deviation) and the fit of a statistical function are represented.

relevant model for studying flow sensors, in particular for terrestrial insects.

Flow sensing has been particularly well studied in the context of startle behavior in crayfish. We cannot do justice here to the many studies done on this system but we can highlight a single recent and interesting aspect. Bidirectional hydrodynamic receptor sensilla are found on the antennules and uropods. Vertical feathered hairs on the antennules are positioned distally on each segment. The feathered hairs are sensitive to water movements as small as $0.02 \mathrm{~mm}$ (57). How do crayfish with their long antennules compute an incoming signal? Crayfish display appropriate and coordinated rapid responses to an incoming source of danger through a mechanism that allows the arrival of sensory-afferent impulses from various distances to be synchronized (57). This mechanism operates via a position-dependent linear gradient of sensory axonal conduction velocities. The axons of the most distal receptors are therefore bigger than those of the most proximal receptors. Studies should be undertaken 
Turbulence: complex flows developing random chaotic motion over a wide range of length scales

Shear flow: a flow in which the velocity varies principally in the direction at right angles to the flow to determine if this morphology-based system exists in insects.

Crayfish are large animals. Flow sensing is also used by much smaller copepods, which make up about $80 \%$ of the mesozooplankton in the oceans and thus serve as an appropriate model system to analyze hydrodynamic signals in water $(41,42,79)$. Kiorboe's latest monograph (46) provides an excellent overview. The interaction between the unsteady flow structure due to many modes of displacement displayed in rapid sequence (free sinking, partial sinking, hovering, vertical swimming upward, and horizontal swimming backward or forward) and the body enables a copepod to sample water efficiently (42). This is reinforced by the rhythmic jumps that copepods make, many times their body size. Jumping thus enables them to change their sampling environment; indeed, they would otherwise always encounter the same particles, owing to recirculating vortices (32). These self-created flow structures, of the order of a body length to a few body lengths and lasting up to tens of seconds, interact with the small-scale turbulence of the ocean, including vortices, shear flow, stagnation points, and jet streams.

\section{PARTICLE IMAGE VELOCIMETRY}

Particle image velocimetry (PIV) is increasingly used for full-field studies of flow around complex geometric shapes and movements. Often, but not necessarily, based on lasers, this technique relies on the illumination of a portion of the fluid (a light sheet in classical PIV), in which inert particles act as markers, as smoke does in classical techniques for visualizing fluid flow, and on highspeed video recording techniques. Micro-PIV is a microscopic technique that operates only in water, as fluorescent markers are used to avoid the backlight from the illuminated object. Although widely used in recent studies of microfluids using channels of depths of a few dozen microns, its use with insects is difficult, not least owing to the scaling necessary for working in water and the relatively big structures to be studied. In planar laser-induced fluorescence, the fluorescent dye emits light in the yellow-orange wavelengths, with intensity proportional to dye concentration when excited by the light from the laser.
Copepods have long antennules with many mechanosensory setae, some as long as $1 \mathrm{~mm}$. Copepods are small and usually follow the bulk flow. Therefore, the setae do not bend, and thus they do not feel any difference in velocity. This may also be the case when they are being eaten by a large predator. This situation is different from that of a small particle passing by. The signal then has a velocity gradient over the body of the animal, and the setae distort, thereby inducing a response. Congeners may also produce an intermediate distortion of the flow field. The triggering of all setae, thus involving the whole flow field, may trigger an escape reaction, whereas a more localized event-involving the response of only a few setae-may be indicative of the arrival of a potential mate or prey. The flow field around moving plankton has been studied using particle image velocimetry (11, $22,70,78)$ to extract velocity profiles, attenuation coefficients, and a sensory field. Using the previously described equation for Stokes creeping flow around a sphere, together with a dipole model for flow at distances far from the body, Kiorboe (46) computed the deformation rate and fluid velocity as a function of the distance from a copepod. He showed that the predicted reaction distance closely matched the reaction distances observed in several biotests and concluded that copepods respond to deformation, with a critical threshold deformation rate of $1-10 \mathrm{~s}^{-1}$. Yen et al. (87) used neurophysiological techniques to demonstrate that a $10-\mathrm{nm}$ deflection in setae was the minimum displacement necessary to initiate a neural response. For 1$\mathrm{mm}$ setae, this displacement corresponds to a threshold of $10^{-5}$ radians.

A fish attacking a copepod appears to use locomotion reminiscent of one of the two foraging modes described for spiders (10). When they perceive a copepod, fish larvae decelerate and approach the prey slowly (58), at a speed that is too low to generate a hydrodynamic signal. The maximal deformation rate produced by small planktonic predators in water is of the order of $10 \mathrm{~s}^{-1}$; effective strategies of escape must therefore be at lower rates. This is indeed observed, with a mean value for prey escape 
rates around $5 \mathrm{~s}^{-1}$. Lower critical rates are more advantageous to the prey. It may be surprising therefore that copepods do not use the limit of the neurophysiological threshold for escaping. A study suggests that this is due to the significant disruption of their feeding behavior (28). This issue needs further consideration; indeed, a life-saving move is far more beneficial than an extra meal, rendering the cost of a false alarm relatively low. An alternative explanation could be linked to the long-lasting effects in the water after escaping violently, which could alert other predators or be used by the same predator. These observations of the aquatic world of crustaceans show that flow-sensing terrestrial arthropods often obey the same rules as aquatic arthropods and they have thus developed similar solutions.

\section{SAND FLOW: FLOW-SENSING MECHANISMS AND USE BY INSECTS}

Could the strong analogies between sensing air flow by insects and sensing water flow by crustaceans be extended to flow sensing by insects in granular matter such as sand? Sand flows like liquids but packs like solids. A crosssection through an avalanche identifies three regions corresponding to three different flow regimes: a solid region under the avalanche in which the grains do not move, or creep slowly; a liquid region in which a dense layer flows; and even a gaseous region in which the grains bounce in all directions, creating a dilute chaotic medium (29). Thus, as a granular medium, sand is neither completely solidlike nor completely liquid-like in its behavior (56). Insects living in sand belong to several orders (e.g., Coleoptera, Neuroptera, Diptera). For example, desert tenebrionids bury themselves $5 \mathrm{~cm}$ below the surface and still hear congeners moving at the surface (36). However, ant lions (Myrmeleontidae) are the sand experts, using its liquid-like and solid-like behavior in ways that are either unknown or undescribed for other animals. We therefore focus on this insect for the remainder of the review. Soil behaves similarly to sand in many respects but is much more complex. Many more orders of insects are found in the top layer of soil, which is partially a granular material. However, we did not find any available data related to flow sensing in soil for insects.

\section{Sand Flow: Its Use by Ant Lions for Prey Capture}

How sand and other particulate matters flow is only partially understood, despite their huge domains of application (blocked flows in silos, the gravel industry or food industry). A sand pile has a given maximal angle at rest, the angle of repose. This is a threshold angle of $\sim 35^{\circ}$, defining a critical state of the granular structure. Inclination at a steeper angle triggers a flow in sheet form, with only a certain portion of the pile starting to move. This state is unstable: Any small perturbation might lead to the formation of an avalanche. In principle, the addition of a single grain is sufficient to cause this. In real life, struggling prey do not always trigger avalanches, and ant lions can provoke avalanches themselves, either by moving within the sand or by throwing sand grains on a struggling prey, with high precision (26). Physicists have shown that the addition of a few grains of sand onto a slope ending against a wall or at the bottom of a pit does not easily trigger avalanches. This is because the first grains, at the front of the avalanche, are building up a small step. This process is repeated, leading to inverse waves moving upward. The critical slope of a pit is thus steeper and more stable than that of a heap (23). The principal aim of an ant lion is not so much to trigger an avalanche as to destabilize its prey.

Avalanches are beneficial for prey capture, but the pit has to be renewed each time. Therefore, any avalanche triggered in the absence of prey is costly, as it leads to pit repair. Of course, as the trap is designed to be in a critical state, avalanches and repair are major activities in an ant lion's daily life. Given the long struggles and violent thrashing of the ant lion larvae, long repairing sessions are needed to ensure that 
the pit is in its optimal state for the next prey (25). Ant lions can determine whether the pit is back to its critical state through further flowsensing mechanisms. For effective restoration of their pits, ant lions use the natural segregation of granular solids of different sizes. The avalanches sort the sand grains, with the larger grains at the bottom of the avalanche. When restoring the pit, ant lions throw away grains of various sizes. Lucas (51) showed that the larger grains go farther, the small particles stopped owing to Stokes law: The higher the drag force due to air relative to the particle momentum, the shorter the distance it will travel. This relationship is sometimes obvious in the wild. Larger rocks are catapulted far outside the pit, which then consists of a more homogeneous group of fine grains.

\section{Wave Propagation in Sand, Flow Sensing, and Orientation in Sand}

Knowledge of wave propagation at the sand surface and subsurface has significantly advanced over recent years. Studies by physicists ( 6 and references therein) confirmed the initial results, obtained by biologists, of the low speed of propagation of the surface waves, or Rayleigh waves, of $\sim 50 \mathrm{~m} \mathrm{~s}^{-1}$ at $100-500 \mathrm{~Hz}$, which is far lower than those observed for glass $\left(\sim 5000 \mathrm{~m} \mathrm{~s}^{-1}\right)$. They showed additionally that the speed of travel is a function of depth. Gravity induces a heterogeneous pressure field in sand, which acts as a wave guide. The highest amplitudes are found at some distance beneath the surface, and the relative vertical and horizontal movements of sand grains also arise as a function of depth.

These new findings have significant implications for the kinds of signals transmitted and received by sand-inhabiting insects. How do insects orientate themselves using flow sensors in such complex systems? Insects such as the ant lion are small in relation to the wavelength of the Rayleigh waves. In such conditions, orientation would seem particularly challenging, given that many orientation mechanisms are dependent on the difference of amplitude and phase of waves arriving at certain places on the body such as legs. Such differences might not exist, or they might be too small. Various measurement points over the whole body would therefore seem to be a better solution. Fascinating studies have shown, however, that minute thermal differences can substantially change the force network within the granular material (25 and references therein). Thus, the directivity of the source could get lost easily, with only a dense network of sensilla producing a reliable threedimensional interpretation of the flow field. Although little is known about the organ, sensilla, or the mechanisms allowing ant lions to perceive avalanches, biotests using artificial prey have shown that they orient themselves and throw sand at the target with high precision (26). Thus, the mechanisms underlying the orientation and flow sensing of small insects in sand remain unclear; indeed, a complete understanding of how sand flows under natural conditions is first needed.

\section{IDENTIFIED GAPS}

We highlight two major issues that must be addressed before major advances, ranging from biomechanics to ecology, can be made in the topics covered in this review.

\section{Beyond Hairs: From Single Mechanosensors to Composite Mechanosystems}

Many ultrastructural studies show that mechanosensors can be categorized into groups or pairs of dissimilar sensors, hairs often classified as levers. Clear examples of the latter include feathered hairs coupled to smooth hairs in crayfish, or insect filliform hairs coupled to campaniforms at their base (2, $3,19,59$, and references therein). In these systems, both sensors operate at different ranges of stimulus amplitude and are mechanically linked through cuticle deformation. In crayfish, long smooth hairs-innervated by many cells and most likely primarily chemoreceptorsstand upright in the boundary layer and possibly above. The mechanoreceptor at its 
base detects the cuticle deflection when the flagellum is moving. The movement of the flagellum triggers a displacement of the relative position of its segments, of feather sensilla at the interface of two segments, and of the cuticle surrounding it, where the smooth hairs and their mechanoreceptors are located. This succession of sensors, each operating within a specific range, is reminiscent of the cricket hair-campaniform sensilla complex. In this complex, larger filiform hairs are morphologically and functionally coupled with campaniform sensilla located at their base, forming a composite mechanosystem. The filiform hairs respond to the slightest hair movements, of the order of $0.1 \mathrm{~mm} \mathrm{~s}^{-1}$. The campaniform sensilla require stronger stimuli, of at least one order of magnitude higher ( $2 \mathrm{~mm} \mathrm{~s}^{-1}$ and more), which cause the hair shaft to displace the socket. Sockets are tilted more easily toward the tip than toward the base of the cercus, suggesting that the membranous zone surrounding each socket is asymmetrically deformable (and that they have a specific preexisting tension) (35). Consequently, the directional response of campaniform sensilla is simpler than that of the associated hair. However, there is a price to pay, albeit a potentially small one, as the much higher amplitude needed to trigger the campaniform sensilla implies that the danger is more than imminent.

The extent of cuticle deformation is therefore of major importance, even with respect to single sensors. The mechanical properties of the complex structure at the base of the hair, the hair socket, and its membrane therefore deserve particular attention, given that they determine the restoration and spring constants of the hair. Microindentation and X-rays (55) are new tools that are particularly appropriate for measuring the Young's modulus and other parameters determining the viscoelastic behavior of the cuticle. A systems approach is needed to understand the joint workings of multiple sensors, even more so if they are both dissimilar in their design but dependent on each other's function.

\section{Sensory Ecology}

The organisms discussed in this review often have no particularity, in terms of hair morphology for flow sensing, behavioral responses to flow, or the physics of flow. Their commonality is the ease of keeping the organism in culture, which bears a hidden price. For these species, few data obtained using ecological conditions in the field are available $(15,16,81)$. We have highlighted here the need for ecologically relevant studies. Further efforts should be made to measure the flow-sensing performance of freely behaving arthropods subjected to realistic stimulus patterns in natural, usually turbulent, environments. For example, several studies suggest that water motion is likely to increase the complexity of the predator-prey relationship under natural conditions. Mann et al. (54) point out that, whereas there is evidence suggesting rapid sensing of local flow conditions, it is uncertain how rapidly microorganisms reorient in a turbulent flow so that they can successfully catch their prey or escape their predators. Similarly, Clarke et al. (13) suggest that turbulence increases the chances of success of a coral fish capturing copepods by interfering with the hydrodynamic sensing of the approaching predator. Determining whether predators are more successful at capturing their prey under turbulent than still flow conditions remains an exciting field of research. Transportable technology and the need to consider relevant stimuli force us to put our boots on, go out, and study the neuromechanics of flow sensing in the most natural conditions possible or, alternatively, to create an artificial world in the lab.

Given the benefits of studying organisms under extreme conditions for understanding their evolution, it may be of interest to study more unusual species in more extreme flow conditions, such as in caves or torrents. Cave habitats often shelter high densities of crickets and large arthropod predators (e.g., amblypigids); attack sequences can thus be frequently and easily observed. The relatively low taxonomic richness and low trophic complexity encountered in caves, in comparison to other 
types of habitats, increase the probability of trophic specialization and therefore of developing highly specialized senses.

\section{TOWARD A GENERALIZED FRAMEWORK FOR FLOW SENSING IN ANY ARTHROPOD}

A direct approach to the study of hair-based flow sensing in arthropods in both air and water is now needed, given that the similarities are striking. Some differences are also notable, at least partly, if not mainly, owing to the fluid characteristics. This call to "look beyond one's pond," as eloquently cited by G.E. Hutchinson in his 1951 paper "Copepodology for the Ornithologist" $(39,66)$, is not new. Indeed, this notion in terms of hair-based flow sensing was actively followed during the 1980s and 1990s by T. Shimozawa (68), who worked both on the cricket's cercal system and on the crayfish's caudal escape system, and by J. Tautz (74), who worked on the early warning system of caterpillars and on the crayfish's caudal escape system. In the past decade, J. Humphrey (38) theoretically analyzed in depth the analogies and differences in single-hair biomechanics in air and water. As a result, this field of research has the advantage of having an unusually well-founded theoretical basis for the fluid forces acting on the hairs and their responses, designed to be applicable in both environments.
Hair-based flow sensing is indeed one of the few areas in which applied mathematics has been the common language of groups as diverse as field ecologists, materials scientists, aerodynamicists, theoretical physicists, computer scientists, and neurobiologists, often funded by the technological branch-and not necessarily the biologically oriented ones-of different research organizations (e.g., the Future and Emergent Technology branch of the Information Society Technologies program in the European Union, or the Defense Advanced Research Projects Agency in the United States). The mathematical toolbox, and its wide acceptance throughout the scientific community, is an unusual asset in sensory ecology. The construction of biomimetic microelectronic mechanical systems (MEMS) based on the design of natural cricket hairs by engineers (see Reference 49 for an example based on cricket hairs) offers a unique opportunity to understand biological principles by tinkering with physical models. Furthermore, it provides a technological showcase for organismal biology, through its impact on bionics and biomimetism. Provided that the current interest in this field can be sustained, we can expect that these opportunities will be seized and that our understanding of hair-based flow sensing will be much further advanced, if not fully elucidated for single hairs, within a decade-ideally for both copepods foraging for food particles in the open ocean as well as crickets escaping lunging predators in the leaf litter.

\section{SUMMARY POINTS}

1. Arthropods carry out flow sensing in various contexts using mechanosensory hairs.

2. The wide variety in hair length, number, and spatial arrangement can be explained partly by the physics of the medium and its interactions with hair arrays.

3. Emergent properties associated with viscous coupling between hairs and array effects may explain the density of hairs in many arthropods.

4. Flow sensing in insects living in sand and soil may follow the same principles as those observed in fluid environments, and thus provide a useful study model.

5. Research on flow sensing in crustaceans and terrestrial arthropods tends to be based on studies carried out independently but converges toward consistent results. 
6. A direct approach is needed to address hair-based fluid flow sensing for arthropods living in all media, using the unique combination of a workable mathematical model and MEMS technology to enable physical modeling at similar scales.

\section{DISCLOSURE STATEMENT}

The authors are not aware of any affiliations, memberships, funding, or financial holdings that might be perceived as affecting the objectivity of this review.

\section{ACKNOWLEDGMENTS}

We thank F. Barth, D. Fields, H. Jiang, J. Miller, B. Statzner, M. Weissburg and J. Yen for sending us their latest works, and L. Bonneau, D. Mellon, J. Humphrey, and T. Steinmann for discussions. This work is part of the research conducted within the Cricket Inspired perCeption and $\mathrm{Au}-$ tonomous Decision Automata (CICADA) project (IST-2001-34718) and within the Customized Intelligent Life Inspired Arrays (CILIA) project (FP6-IST-016039). Both projects are funded by the European Community under the Information Society Technologies (IST) Program, Future and Emergent Technologies (FET), Lifelike Perception Systems action. We apologize to the many scientists whose works were not cited, or cited only too briefly, owing to space constraints.

\section{LITERATURE CITED}

1. Acebes A, Cobb M, Ferveur JF. 2003. Species-specific effects of single sensillum ablation on mating position in Drosophila. 7. Exp. Biol. 206:3095-100

2. Barth FG. 2002. A Spider's World: Senses and Behavior. Berlin: Springer. 394 pp.

3. Barth FG. 2004. Spider mechanoreceptors. Curr. Opin. Neurobiol. 14:415-22

4. Bathellier B, Barth FG, Albert JT, Humphrey JA. 2005. Viscosity-mediated motion coupling between pairs of trichobothria on the leg of the spider Cupiennius salei. 7. Comp. Physiol. A 191:733-46

5. Bergman DA, Redman CN, Fero KC, Simon JL, Moore PA. 2006. The impacts of flow on chemical communication strategies and fight dynamics of crayfish. Mar. Freshw. Behav. Physiol. 39:245-58

6. Bonneau L, Andreotti B, Clement E. 2008. Evidence of Rayleigh-Hertz surface waves and shear stiffness anomaly in granular media. Phys. Rev. Lett. 101:118001

7. Boxshall GA, Huys R. 1998. The ontogeny and phylogeny of copepod antennules. Philos. Trans. R. Soc. London B 353:765-86

8. Bundy MH, Paffenhöfer GA. 1996. Analysis of flow fields associated with freely swimming calanoid copepods. Mar. Ecol. Prog. Ser. 133:99-113

9. Camhi J. 1984. Neuroethology: Nerve Cells and the Natural Behavior of Animals. Sunderland, MA: Sinauer. $432 \mathrm{pp}$.

10. Casas J, Steinmann T, Dangles O. 2008. The aerodynamic signature of running spiders. PLoS ONE 3:e2116

11. Catton KB, Webster DR, Brown J, Yen J. 2007. Quantitative analysis of tethered and free-swimming copepodid flow fields. F. Exp. Biol. 210:299-310

12. Cheer AYL, Koehl MAR. 1987. Paddles and rakes: fluid flow through bristled appendages of small organisms. 7. Theor. Biol. 129:17-39

13. Clarke RD, Buskey EJ, Marsden KC. 2005. Effects of water motion and prey behavior on zooplankton capture by two coral reef fishes. Mar: Biol. 146:1145-55

14. Cummins B, Gedeon T, Klapper I, Cortez R. 2007. Interaction between arthropod filiform hairs in a fluid environment. F. Theor. Biol. 247:266-80

\section{Integrative biology at} its best.

10. First quantification of the upstream flow created by a running predatory arthropod and used by its prey. 
26. Orientation in sand.

\section{The latest} neurophysiological results on flow sensing in copepods.
38. The biomechanics of hair-based flow sensing masterfully reviewed.
15. Dangles O, Casas J, Coolen I. 2006. Textbook cricket goes to the field: the ecological scene of the neuroethological play. F. Exp. Biol. 209:393-98

16. Dangles O, Irschick D, Chittka L, Casas J. 2009. Variability in sensory ecology: expanding the bridge between physiology and evolutionary biology. Q. Rev. Biol. 84:51-74

17. Dangles O, Magal C, Pierre D, Olivier A, Casas J. 2005. Variation in morphology and performance of predator-sensing system in wild cricket populations. F. Exp. Biol. 208:461-68

18. Dangles O, Ory N, Steinmann T, Christides JP, Casas J. 2006. Spider's attack versus cricket's escape: velocity modes determine success. Anim. Behav. 72:603-10

19. Dangles O, Pierre D, Magal C, Vannier F, Casas J. 2006. Ontogeny of air-motion sensing in cricket. 7. Exp. Biol. 209:4363-70

20. Dangles O, Steinmann T, Pierre D, Vannier F, Casas J. 2008. Relative contributions of organ shape and receptor arrangement to the design of cricket's cercal system. F. Comp. Physiol. A 194:653-63

21. Denissenko P, Lukaschuk S, Breithaupt T. 2007. The flow generated by an active olfactory system of the red swamp crayfish. F. Exp. Biol. 210:4083-91

22. Doall MH, Strickler JR, Fields DM, Yen J. 2002. Mapping the free-swimming attack volume of a planktonic copepod, Euchaeta rimana. Mar. Biol. 140:871-79

23. Duran J. 2000. Sands, Powders, and Grains: An Introduction to the Physics of Granular Materials. New York: Springer. 214 pp.

24. Dusenberry D. 1992. Sensory Ecology: How Organisms Acquire and Respond to Information. New York: Freeman. 558 pp.

25. Fertin A, Casas J. 2006. Efficiency of antlion trap construction. F. Exp. Biol. 209:3510-15

26. Fertin A, Casas J. 2007. Orientation towards prey in antlions: efficient use of wave propagation in sand. F. Exp. Biol. 210:3337-43

27. Fields DM, Weissburg MJ. 2004. Rapid firing rates from mechanosensory neurons in copepod antennules. F. Comp. Physiol. A 190:877-82

28. Fields DM, Yen J. 1997. The escape behavior of marine copepods in response to a quantifiable fluid mechanical disturbance. 7. Plankton Res. 19:1289-304

29. Forterre Y, Pouliquen O. 2008. Flows of dense granular media. Annu. Rev. Fluid Mech. 40:1-24

30. French A. 2009. The systems analysis approach to mechanosensory coding. Biol. Cybern. 100:417-26

31. Gayev YA, Hunt CR, eds. 2007. Flow and Transport Processes with Complex Obstructions. Dordrecht, The Netherlands: Springer. 414 pp.

32. Genin A, Jaffe JS, Reef R, Richter C, Franks PJS. 2005. Swimming against the flow: a mechanism of zooplankton aggregation. Science 308:860-62

33. Gnatzy W. 1996. Digger Wasp vs Cricket: Neuroethology of a Predator-Prey Interaction. Stuttgart, Ger.: Fischer. $92 \mathrm{pp}$.

34. Hart DD, Finelli CM. 1999. Physical-biological coupling in streams: the pervasive effects of flow on benthic organisms. Annu. Rev. Ecol. Syst. 30:363-95

35. Heußlein R, Gras H, Gnatzy W. 2009. Functional coupling of cercal filiform hairs and campaniform sensilla in crickets. In Functional Surfaces in Biology. Vol. 1: Small Structures with Big Effects, ed. SN Gorb. Dordrecht, The Netherlands: Springer. In press

36. Hill PSM. 2008. Vibrational Communication in Animals. Cambridge, MA: Harvard Univ. Press. 272 pp.

37. Horsmann U, Heinzel HG, Wendler G. 1983. The phasic influence of self-generated air current modulations on the locust flight motor. 7. Comp. Physiol. A 150:427-38

38. Humphrey JA, Barth FG. 2008. Medium flow-sensing hairs: biomechanics and models. In $A d$ vances in Insect Physiology. Vol. 34: Insect Mechanics and Control. ed. J Casas, SJ Simpson, pp. 1-80. Amsterdam, The Netherlands: Elsevier. 396 pp.

39. Hutchinson GE. 1951. Copepodology for the ornithologist. Ecology 32:571-77

40. Jacobs GA, Miller JP, Aldworth Z. 2008. Computational mechanisms of mechanosensory processing in the cricket. F. Exp. Biol. 211:1819-28

41. Jiang H, Paffenhöfer G. 2008. Hydrodynamic signal perception by the copepod Oithona plumifera. Mar. Ecol. Prog. Ser. 373:37-52

42. Jiang H, StricklerJR. 2007. Copepod flow modes and modulation: a modelling study of the water currents produced by an unsteadily swimming copepod. Philos. Trans. R. Soc. London B 362:1959-71 
43. Joanidopoulos KD, Marwan W. 1999. Neural integration of chemosensory and mechanosensory stimuli triggers the mating response in males of the giant rotifer Asplanchna sieboldi. Ethology 105:465-75

44. Kaiser P. 1965. Uber Netzbau und Stroemungssin bei den Larven der Gattung Hydropsyche PICT. (Ins., Trichoptera). Int. Rev. Ges. Hydrobiol. Hydrogr: 50:169-224

45. Kant R, Humphrey JA. 2009. Response of cricket and spider motion-sensing hairs to airflow pulsations. 7. R. Soc. Interface. doi:10.1098/rsif.2008.0523

46. Kiorboe T, ed. 2008. A Mechanistic Approach to Plankton Ecology. Princeton, NJ: Princeton Univ. Press. 209 pp.

47. Koehl MA. 1996. When does morphology matter? Annu. Rev. Ecol. Syst. 27:501-42

48. Koehl MA, KoseffJR, Crimaldi JP, McCay MG, Cooper T, et al. 2001. Lobster sniffing: antennule design and hydrodynamic filtering of information in an odor plume. Science 294:1948-51

49. Krijnen GJM, Dijkstra M, van BaarJJ, Shankar SS, Kuipers WJ, et al. 2006. MEMS based hair flow-sensors as model systems for acoustic perception studies. Nanotechnology 17:84-89

50. Legier-Visser M, Mitchell JG, Okubo A, Fuhrman JA. 1986. Mechanoreception in calanoid copepods: a mechanism for prey detection. Mar. Biol. 90:529-35

51. Lucas J. 1982. The biophysics of pit construction by antlion larvae (Myrmeleon, Neuroptera). Anim. Behav. 30:651-64

52. Ludowiak K, Weeks J. 2008. Neurosensory ecology. 7. Exp. Biol. 211:1705-828

53. Magal C, Dangles O, Caparroy P, Casas J. 2006. Hair canopy of cricket sensory system tuned to predator signals. 7. Theor. Biol. 241:459-66

54. Mann J, Ott S, Pecseli HL, Trulsen J. 2006. Laboratory studies of predator-prey encounters in turbulent environments: effects of changes in orientation and field of view. F. Plankton Res. 28:509-22

55. McConney ME, Schaber CF, Julian MD, Eberhardt WC, Humphrey JA, et al. 2008. Surface force spectroscopic point load measurements and viscoelastic modelling of the micromechanical properties of air flow sensitive hairs of a spider (Cupiennius salei). 7. R. Soc. Interface 6:681-94

56. Mehta A. 2007. Granular Physics. Cambridge, UK: Cambridge Univ. Press. 305 pp.

57. Mellon D, Christison-Lagay K. 2008. A mechanism for neuronal coincidence revealed in the crayfish antennule. Proc. Natl. Acad. Sci. USA 105:14626-31

58. Munk P, Kiorboe T. 1985. Feeding-behavior and swimming activity of larval herring (Clupea harengus) in relation to density of copepod nauplii. Mar. Ecol. Prog. Ser. 24:15-21

59. Murray JA, Estepp J, Cain SD. 2006. Advances in the neural bases of orientation and navigation. Integr: Comp. Biol. 46:871-79

60. North G, Greenspan RJ, eds. 2007. Invertebrate Neurobiology. Cold Spring Harbor, NY: Cold Spring Harb. Lab. Press. 800 pp.

61. Patria MP, Wiese K. 2004. Swimming in formation in krill (Euphausiacea), a hypothesis: dynamics of the flow field, properties of antennular sensor systems and a sensory-motor link. F. Plankton Res. 26:1315-25

62. Prete FR, ed. 2004. Complex Worlds from Simpler Nervous Systems. Cambridge, MA: MIT Press. 456 pp.

63. Rieke F, Warland D, de Ruyter van Steveninck R, Bialek W. 1997. Computational Neuroscience: Spikes: Exploring the Neural Code. Cambridge, MA: MIT Press. 416 pp.

64. Roeder KD. 1948. Organization of the ascending giant fiber system in the cockroach (Periplaneta americana L.). 7. Exp. Zool. 108:243-62

65. Sane SP, Dieudonné A, Willis MA, Daniel TL. 2007. Antennal mechanosensors mediate flight control in moths. Science 315:863-66

66. Schminke HK. 2007. Entomology for the copepodologist. F. Plankton Res. 29(Suppl. 1):149-62

67. Sherry DF. 2006. Neuroecology. Annu. Rev. Psychol. 57:167-97

68. Shimozawa T, Murakami J, Kumagai T. 2003. Cricket wind receptors: thermal noise for the highest sensitivity known. In Sensors and Sensing in Biology and Engineering, ed. FG Barth, JA Humphrey, TW Secomb, pp. 145-59. Berlin: Springer. 399 pp.

69. Smith CUM. 2000. Biology of Sensory Systems. Hoboken, NJ: Wiley. 445 pp.

70. Stamhuis EJ, Videler JJ, van Duren LA, Muller UK. 2002. Applying digital particle image velocimetry to animal-generated flows: traps, hurdles and cures in mapping steady and unsteady flow in $R e$ regimes between $10^{-2}$ and $10^{5}$. Exp. Fluids 33:801-13
55. Material characterization with latest technology is revitalizing functional morphology.

68. Outstanding account of the extreme sensitivity of mechanosensory hairs. 
71. A bird of prey point of view by the pioneer of laser-based flow measurements in a seminatural aquatic environment at the scale relevant to insects.

76. Far-ranging implications, at the community level, of hair-based flow sensing by one of the pioneers.

79. The principles of the microphysics of flow in the life of copepods.
71. Statzner B. 2008. How views about flow adaptations of benthic stream invertebrates changed over the last century. Int. Rev. Hydrobiol. 93:593-605

72. Steinmann T, Casas J, Krijnen G, Dangles O. 2006. Air-flow sensitive hairs: boundary layers in oscillatory flows around arthropod appendages. F. Exp. Biol. 209:4398-408

73. Suter RB. 2003. Trichobothrial mediation of an aquatic escape response: directional jumps by the fishing spider, Dolomedes triton, foil frog attacks. F. Insect Sci. 3:19

74. Tautz J. 1989. Medienbewegung in der Sinneswelt des Artbropoden. Fallstudien zu einer Sinnesökologie. Stuttgart, Ger.: Fischer. 59 pp.

75. Tautz J, Markl H. 1978. Caterpillars detect flying wasps by hairs sensitive to airborne vibration. Behav. Ecol. Sociobiol. 4:101-10

76. Tautz J, Rostas M. 2008. Honeybee buzz attenuates plant damage by caterpillars. Curr. Biol. 18:R1125-26

77. Triblehorn JD, Yager DD. 2006. Wind generated by an attacking bat: anemometric measurements and detection by the praying mantis cercal system. F. Exp. Biol. 209:1430-40

78. van Duren LA, Videler JJ. 2003. Escape from viscosity: the kinematics and hydrodynamics of copepod foraging and escape swimming. 7. Exp. Biol. 206:269-79

79. Visser AW. 2001. Hydromechanical signals in the plankton. Mar. Ecol. Prog. Ser. 222:1-24

80. Weatherby TM, Lenz PH. 2000. Mechanoreceptors in calanoid copepods: designed for high sensitivity. Artbropod Struct. Dev. 29:275-88

81. Weissburg MJ, Browman HI. 2005. Sensory biology: linking the internal and external ecologies of marine organisms. Mar. Ecol. Prog. Ser. 287:263-65

82. Weissburg MJ, Zimmer-Faust RK. 1994. Odor plumes and how blue crabs use them in finding prey. 7. Exp. Biol. 197:349-75

83. Weygoldt P. 2000. Whip Spiders. (Chelicerata: Amblypygi). Their Biology, Morphology and Systematics. Stenstrup, Denmark: Apollo Books. 163 pp.

84. Willis MA, Avondet JL, Finnell AS. 2008. Effects of altering flow and odor information on plume tracking behavior in walking cockroaches, Periplaneta americana (L.). 7. Exp. Biol. 211:2317-26

85. Wright J, Boxshall AJ. 1999. The influence of small-scale flow and chemical cues on the settlement of two congeneric barnacle species. Mar. Ecol. Prog. Ser. 183:179-87

86. Wyeth RC, Woodward OM, Willows AO. 2006. Orientation and navigation relative to water flow, prey, conspecifics, and predators by the nudibranch mollusc Tritonia diomedea. Biol. Bull. 210:97-108

87. Yen J, Lenz PH, Gassie DV, Hartline DK. 1992. Mechanoreception in marine copepods: electrophysiological studies on the first antennae. F. Plankton Res. 14:495-512

88. Yen J, Strickler JR. 1996. Advertisement and concealment in the plankton: What makes a copepod hydrodynamically conspicuous? Invertebr: Biol. 115:191-205 
(

\section{Contents}

Annual Review of

Entomology

Volume 55, 2010

Frontispiece

Mike W. Service ........................................................... xiv

The Making of a Medical Entomologist

Mike W. Service .................................................................. 1

Ecology of Herbivorous Arthropods in Urban Landscapes

Michael F. Raupp, Paula M. Shrewsbury, and Daniel A. Herms .......................19

Causes and Consequences of Cannibalism in Noncarnivorous Insects

Matthew L. Richardson, Robert F. Mitchell, Peter F. Reagel,

and Lawrence M. Hanks....

Insect Biodiversity and Conservation in Australasia

Peter S. Cranston

Ekbom Syndrome: The Challenge of "Invisible Bug" Infestations

Nancy C. Hinkle .....

Update on Powassan Virus: Emergence of a North American

Tick-Borne Flavivirus

Gregory D. Ebel

Beyond Drosophila: RNAi In Vivo and Functional Genomics in Insects

Xavier Bellés

Dicistroviruses

Bryony C. Bonning and W. Allen Miller

Olive Fruit Fly: Managing an Ancient Pest in Modern Times

Kent M. Daane and Marshall W. Fohnson

Insect Silk: One Name, Many Materials

Tara D. Sutherland, James H. Young, Sarab Weisman, Cheryl Y. Hayashi,

and David 7. Merritt

Bayesian Phylogenetics and Its Influence on Insect Systematics

Fredrik Ronquist and Andrew R. Deans

Insect Fat Body: Energy, Metabolism, and Regulation

Estela L. Arrese and Fose L. Soulages 
Sex Differences in Phenotypic Plasticity Affect Variation in Sexual Size

Dimorphism in Insects: From Physiology to Evolution

R. Craig Stillwell, Wolf U. Blanckenhorn, Tiit Teder, Goggy Davidowitz,

Charles W. Fox

Facultative Symbionts in Aphids and the Horizontal Transfer of

Ecologically Important Traits

Kerry M. Oliver, Patrick H. Degnan, Gaelen R. Burke, and Nancy A. Moran

Honey Bees as a Model for Vision, Perception, and Cognition

Mandyam V. Srinivasan

Invasion Biology, Ecology, and Management of the Light Brown Apple

Moth (Tortricidae)

D.M. Suckling and E.G. Brockerhoff

Feeding Mechanisms of Adult Lepidoptera: Structure, Function, and

Evolution of the Mouthparts

Harald W. Krenn

Integrated Management of Sugarcane Whitegrubs in Australia:

An Evolving Success

Peter G. Allsopp

The Developmental, Molecular, and Transport Biology of Malpighian

Tubules

Klaus W. Beyenbach, Helen Skaer, and Julian A.T. Dow

Biorational Approaches to Managing Stored-Product Insects

Thomas W. Phillips and Fames E. Throne

Parallel Olfactory Systems in Insects: Anatomy and Function

C. Giovanni Galizia and Wolfgang Rössler

Integrative Taxonomy: A Multisource Approach to Exploring

Biodiversity

Birgit C. Schlick-Steiner, Florian M. Steiner, Bernbard Seifert,

Christian Stauffer, Erhard Christian, and Ross H. Crozier

Evolution of Plant Defenses in Nonindigenous Environments

Colin M. Orians and David Ward

Landscape Epidemiology of Vector-Borne Diseases

William K. Reisen

Role of Adhesion in Arthropod Immune Recognition

Otto Schmidt, Kenneth Söderbäll, Ulrich Theopold, and Ingrid Faye

Physical Ecology of Fluid Flow Sensing in Arthropods

férôme Casas and Olivier Dangles 
Managing Invasive Populations of Asian Longhorned Beetle and Citrus

Longhorned Beetle: A Worldwide Perspective

Robert A. Haack, Franck Hérard, Fianghua Sun, and Fean 7. Turgeon

Threats Posed to Rare or Endangered Insects by Invasions of

Nonnative Species

David L. Wagner and Roy G. Van Driesche

Malaria Management: Past, Present, and Future

A. Enayati and 7. Hemingway

Regulation of Midgut Growth, Development, and Metamorphosis

Raziel S. Hakim, Kate Baldwin, and Guy Smagghe

Cellulolytic Systems in Insects

Hirofumi Watanabe and Gaku Tokuda

\section{Indexes}

Cumulative Index of Contributing Authors, Volumes 46-55

Cumulative Index of Chapter Titles, Volumes 46-55

\section{Errata}

An online log of corrections to Annual Review of Entomology articles may be found at http://ento.annualreviews.org/errata.shtml 